\title{
Effects of a Posture Sensing Composite Display Device (PSCD) on Lumbopelvic Motion during Computer Work
}

\author{
SE-YEON PARK ${ }^{1)}$, WON-GYU YOO ${ }^{2)}$ \\ 1) Department of Physical Therapy, The Graduate School, Inje University \\ ${ }^{2)}$ Department of Physical Therapy, College of Biomedical Science and Engineering, Inje University: \\ 607 Obangdong, Gimhae, Gyeongsangnam-do, 621-749 Republic of Korea. \\ TEL: +82 55-320-3994,FAX: +82 55-329-1678,E-mail:won7y@inje.ac.kr
}

\begin{abstract}
Purpose] This study evaluated the efficacy of a newly designed chair, equipped with a Posture Sensing Composite Display Device (PSCD), for correcting poor trunk posture while performing computer work. [Subjects] We recruited 10 male workers who regularly used a computer for at least $8 \mathrm{~h}$ per day. [Methods] Subjects performed computer work while sitting on the newly designed chair with or without the PSCD. Lumbopelvic kinematic data were recorded for $60 \mathrm{~s}$ following 10, 20, and $30 \mathrm{~min}$ of computer work using a three-dimensional motion analysis system. [Results] The newly designed chair equipped with the PSCD significantly reduced lumbar flexion, lateral bending, and rotation during computer work. [Conclusion] The PSCD may prevent work-related musculoskeletal disorders by enabling a worker to maintain an upright posture during computer work.
\end{abstract}

Key words: Poor posture, Posture sensing, Seated worker

(This article was submitted Jul. 28, 2010, and was accepted Sep. 7, 2010)

\section{INTRODUCTION}

Research has shown that ergonomic interventions reduce the incidence and prevalence of work related musculoskeletal disorders, including low back pain and neck pain caused by prolonged computer work $^{1-3)}$. A correct sitting posture adopted by computer workers is associated with an improvement in neck and shoulder pain as well as low back pain ${ }^{4,5}$. In ergonomic interventions for correcting sitting posture, a few studies have reported the positive effect of an ergonomic chair during computer work ${ }^{5-7)}$. Yoo et al. suggested that a modified chair with an auditory biofeedback system was an effective ergonomic intervention for correcting the slumped sitting posture often adopted during computer work $^{5)}$. However, other research using an auditory feedback device showed it to be distracting, and the work efficiency of seated workers was affected. In poor posture, the stereoscopic mobility of the lumbar region need to be considered because lateral bending and rotation of the lumbar segment could be risk factors in low back pain ${ }^{8-10)}$. However, devices for correcting poor posture with stereoscopic consideration have not yet been developed. In this study, we evaluated the efficacy of a newly designed chair equipped with a Posture Sensing Composite Display Device (PSCD) for correcting poor posture by assessing lumbopelvic motion during computer work.

\section{SUBJECTS AND METHODS}

Ten male workers were recruited for this study. Subjects were included if they were regularly seated and used a computer for at least $8 \mathrm{~h}$ per day; they were excluded if they had a history of upper or lower extremity injuries/diseases that could affect their ability to work at a computer. Participants were aged between 24 and 29 years, and had a mean height and weight of $176.1 \pm 5.4 \mathrm{~cm}$ and $64.6 \pm 4.9 \mathrm{~kg}$, respectively.

Ethical approval for this study was obtained from the Inje University Faculty of Health Sciences Human Ethics Committee. The subjects provided their informed consent prior to participation.

Lumbar movements were assessed using a 3-D motion analysis system (Vicon MX; Oxford Metrics, Oxford, UK) with six infrared cameras. Kinematic data from the lumbar spine and pelvis were collected by attaching seven markers to the subject's skin with double-sided tape. Four markers were attached at the lumbar region and defined the lumbar segment; they were placed at L1 and L2 and on the left and right sides of L1 and L2, 1 inch apart. The pelvic segment was defined by markers placed at the midpoint of the posterior superior iliac spine (PSIS) and the anterior superior iliac spine (ASIS), and to the left and right of the ASIS. The kinematic data were collected at a sampling rate of $50 \mathrm{~Hz}$ by Nexus 1.4 (Oxford Metrics) software and 
smoothed using a Woltring filter with a mean squared error of 15. Marker trajectories were reconstructed using the Vicon Bodybuilder (Oxford Metrics, UK) and Euler angles $(\mathrm{X} ; \mathrm{Y} ; \mathrm{Z})$ were produced for the 2 body segments: lumbar and pelvis. Measured values of lumbar flexion and lateral bending and rotation were calculated relative to pelvic segment, using a method modified from a previous study ${ }^{11,12)}$.

The PSCD (Posture Sensing Composite Display Device) consisted of three proximity sensors (HYT-F4R4; HY Technologies, California, USA) with a range of $40 \times 40 \mathrm{~mm}$ attached to the chair by three adjustable bars and connected to a display device. The sensors were located at the back, and on the left and right sides of the chair. They were used to detect lateral bending and rotation of the lumbar segments and their positions were adjusted for each subject. The height of the posterior sensor was set to the level of the spinous process $\mathrm{T} 10 \mathrm{~L} 1$, and the collateral sensors were placed at the horizontal height of the left and right acromions. When the proximity sensors detected posture changes, including slumping, lateral bending, and rotation of the lumbar segments, they provided visual feedback in the form of a red lamp, which highlighted the words "poor posture". The red lamp was placed just beside the computer monitor in the participant's workspace. The subjects were instructed about correct upright sitting posture and told to correct their sitting posture when the red lamp was illuminated. All of the procedures were performed by the same investigator to reduce variability in marker placement and interventions.

All subjects performed selected computer work for 30 min with and without the PSCD. The order of the tests was randomly selected, there was a 30-min interval between the tests, and each subject was allowed a 5-min adjustment period to allow them to adopt a position that they found comfortable for working. We hypothesized that instant maximum or minimum values of the lumbar kinematics would not reflect the overall movement during the computer work. Therefore, average lumber flexion, rotation, and side bending angles were measured with the $3-\mathrm{D}$ motion analysis system to assess the overall changes of trunk movement ${ }^{5}$. Kinematic data were collected for $60 \mathrm{~s}$ following 10, 20, and 30 min of computer work. Values for lumbar kinematic data were expressed as means for statistical analyses.

SPSS version 12.0 (SPSS, Chicago, IL, USA) was used to identify significant differences in flexion, lateral bending, and rotation angles of the lumbar segment. The paired t-test was used to detect the significance of differences in the data collected in the presence and absence of the PSCD. All significance levels were chosen as $\mathrm{p}<0.05$.

\section{RESULTS}

The chair with the PSCD caused subjects to maintain an erect spine posture during computer work. It caused a significant reduction in mean lumbar flexion and coupled motion of lateral bending and rotation during computer work, compared with the chair without the device $(p<0.05$; Table 1).
Table 1. Comparison of the mean values of lumbar flexion, lateral bending, and rotation angles

\begin{tabular}{lcr}
\hline \multirow{2}{*}{ Lumbar angle } & \multicolumn{2}{c}{ Mean $\pm \mathrm{SD}\left({ }^{\circ}\right)$} \\
\cline { 2 - 3 } & Without PSCD & \multicolumn{1}{c}{ With PSCD } \\
\hline Flexion & $27.2 \pm 7.6$ & $11.1 \pm 3.4^{*}$ \\
L. rotation & $15.1 \pm 5.1$ & $9.9 \pm 4.6^{*}$ \\
R. rotation & $18.4 \pm 4.7$ & $12.8 \pm 3.9^{*}$ \\
L. lateral bending & $14.8 \pm 3.5$ & $10.6 \pm 2.7^{*}$ \\
R. lateral bending & $24.5 \pm 4.9$ & $14.7 \pm 3.7^{*}$ \\
\hline
\end{tabular}

*: $\mathrm{p}<0.05$

\section{DISCUSSION}

Low back pain and neck pain are major health problems in the computer workplace ${ }^{1,13}$. Maintaining a slumped sitting posture is a risk factor for work-related muscular disorders and is closely associated with the incidence of low back pain ${ }^{11,14,15)}$. Dolan and Green ${ }^{11)}$ found that a prolonged slumped sitting posture resulted in impaired proprioception of "good posture" and repositioning sense. In the slumped sitting posture, as compared to lumbopelvic sitting, muscular activation of the lumbopelvic region is reduced and body weight is supported by passive structures ${ }^{15}$.

In the present study, we investigated the efficacy of a newly designed chair equipped with a PSCD at correcting poor sitting posture during computer work. The goal of the present study was not to determine a solution or reveal the mechanisms behind musculoskeletal disorders. Instead, we determined whether a posture sensing chair using a visual feedback device had a positive effect on re-education of the standard sitting posture. Previous studies found that computer workers using chairs with simple proximity sensors had decreased forward head, shoulder, and lumbar flexion angles, compared with those using chairs without the devices ${ }^{5}$. However, prolonged rotation of the lumbar posture in the work place is a risk factor for low back pain ${ }^{8)}$. Additionally, lateral flexion of the lumbar spine increases the shearing force of the spinal column and may be a risk factor for scoliosis ${ }^{9,16)}$. Our findings correspond with those of previous reports investigating methods for the prevention of the slumped sitting posture and provide additional advantages because we were able to correct lateral bending and rotation of the lumbar spine.

Within physical therapy intervention, ideal (upright) sitting posture is considered as a position in which the subject has a neutral pelvic tilt and neutral lumbar and thoracic posture ${ }^{15,16)}$. Several studies have suggested that a continuous upright sitting posture prevents low back pain during computer work ${ }^{1,4,5)}$.

Our findings indicated that the PSCD system could be an effective as an ergonomic intervention tool for preventing habitual poor posture by promoting an upright sitting during computer work.

Backrests and armrests are generally known for their ability to decrease low back and neck load during computer work. However, usage of arm rests and a back rest also 
might lead to habitual leaning posture unless they are used properly. Thus, we didn't include a back rest or arm rests in present study.

Our findings indicate that the PSCD system could be an effective as ergonomic intervention tool for preventing habitually poor posture by promoting an upright sitting during computer work.

There are several limitations to our study. Decreased lumbar motion and static upright posture within individual workers could induce muscle fatigue in the lumbar extensor muscles and reduce work efficiency. Our results cannot be generalized because of the small number of subjects and the absence of arm rests and a back rest. Future research must consider these limitations and use composite studies assessing both muscular activation of the lumbar region and lumbopelvic kinematics as well as usage of back and arm rests.

\section{ACKNOWLEDGEMENTS}

This research was supported by Basic Science Research Program through the National Research Foundation of Korea (NRF) funded by the Ministry of Education, Science and Technology (No. 2010-0003874).

\section{REFERENCES}

1) Pillastrini P, Mugnai R, Bertozzi L, et al.: Effectiveness of an ergonomic intervention on work-related posture and low back pain in video display terminal operators: A 3 year cross-over trial. Appl Ergonomics, 2010, 41: 436-443.
2) Straker L, Burgess-Limerick R, Pollock C, et al.: The influence of desk and display design on posture and muscle activity variability whilst performing information technology tasks. Appl Ergonomics, 2009, 40: 852-859.

3) Mork PJ, Westgaard RH: Back posture and low back muscle activity in female computer workers: A field study. Clin Biomech, 2009, 24: 169-175.

4) Caneiro JP, O'Sullivan PB, Burnett A, et al.: The influence of different sitting postures on head/neck posture and muscle activity. Manual Therapy, 2010, 15: 54-60.

5) Yoo WG, Yi CH, Kim MH: Effects of a proximity-sensing feedback chair on head, shoulder, and trunk postures when working at a visual display terminal. J Occup Rehabil, 2006, 16: 631-637.

6) Reeves KB, Stanfield J, Hughes L: Assessment of video display workstation set up on risk factors associated with the development of low back and neck discomfort. Int J Ind Ergon, 2005,35: 593-604.

7) Driessen MT, Proper KI, Tulder MW, et al.: The effectiveness of physical and organisational ergonomic interventions on low back pain and neck pain: a systematic review. Occup Environ Med, 2010, 67: 277-285.

8) Toren A: Muscle activity and range of motion during active trunk rotation in a sitting posture. Applied Ergonomics, 2001, 32: 583-591.

9) Marras WS, Granata KP: Spine loading during trunk lateral bending motions. J. Biomech, 1997, 30: 697-703.

10) Davis KG, Marras WS: The effects of motion on trunk biomechanics. Clin Biomech, 2000, 15: 703-717.

11) Dolan KJ, Green A: Lumbar spine reposition sense: The effect of a 'slouched' posture. Manual Therapy, 2006, 11: 202-207.

12) Chung CY, Park MS, Lee SH, et al.: Kinematic aspects of trunk motion and gender effect in normal adults. J Neuroeng Rehab, 2010, 15: 7-9.

13) Lis AM, Black KM, Korn H, et al.: Association between sitting and occupational LBP. Eur Spine J, 2007, 16: 283-298.

14) Søndergaard KHE, Olesen CG, Søndergaard EK, et al.: The variability and complexity of sitting postural control are associated with discomfort. J. Biomech, 2010, 43: 1997-2001.

15) O'Sullivan PB, Grahamslaw KM, Kendell M, et al.: The effect of different standing and sitting postures on trunk muscle activity in a pain-free population SPINE, 2002, 27: 1238-1244.

16) Neumann DA: Kinesiology of the musculoskeletal system (1st ed). St. Louis: Mosby, 2002, pp.301-303. 